\title{
Alternate Vision Assistance Using Deep Learning
}

\author{
Shameen Basharat ${ }^{1}$, Sapna Jain ${ }^{2}$ \\ \{shameenbasharat@yahoo.com, \\ drsapnajain@jamiahamdard.ac.in\} \\ 1,2 Department of CSE, Jamia Hamdard
}

\begin{abstract}
Visual weakness and visual deficiency brought about by different illnesses has been enormously diminished, yet danger of age-related or intrinsic visual hindrance still hovers.

Visual data is the reason for most acknowled errands, so disabled individuals battle a great deal since they fail to get essential data about the present condition. Performing every day movement can be particularly troublesome; it is difficult for the oblivious in regards to know about environment. With the ongoing advances in innovation it is conceivable to stretch out the help given to individuals with visual debilitation during theirdevelopment.

PC vision advancements, particularly the profound convolutional neural system, have recently evolved. It is promising to utilize the condition of-craftsmanship PC vision strategies to help individuals with vision misfortune. In this exploration venture, I will in general give a continuous article recognition and position estimation pipeline, with the objective of advising the client about encompassing item and their spatial position utilizing binaural sound.

Towards the end, different promising bearings are examined to fill in as rules for future work in the field of object identification and neural system based learning frameworks.
\end{abstract}

Keywords: Visual debilitation, Computer vision innovations, object identification, deep learning, object location structure, CAFFE Model

\section{Introduction}

More than 285 million individuals are outwardly debilitated around the world: 39 million of them being visually impaired and 246 million have low vision (WHO report). Vision impedance is expanding with increasing population. About $90 \%$ of them are living in developing nations. A visually impaired individual is dependent on other individual's data or support. Visually disabled individuals endure a great deal of stress and suffer because of inaccessibility to information to help them arrange their whole day exercises which are of fundamental significance for their wellbeing and prosperity. 


\section{Background}

In the present situation, every one of the individuals who are visually impaired or outwardly tested experience extraordinary difficulties in their everyday life. They are reliant on others for help. To remain refreshed and mindful of what is happening around them or how to manage routine exercises alone, there is a critical need of a well-created framework to support them and make them feel that they are the same as us.

\subsection{The Traditional Object Detection Pipeline}

The principal technique is definitely not an unadulterated start to end object identifier. The subsequent technique to profound learning object discovery anyway allows to treat your preprepared order arrange as a base system in a profound learning object location structure. We can make a total beginning to end efficient learning-based article identifier. On the negative side it requires a touch of personal information on working of these identifiers. There are numerous segments, sub-parts of an efficient learning object finder:

1. The article identification system (ex. Quicker R-CNN, SSD, YOLO).

2. The base system which fits into the article identification structure. Base systems include:

$\mathrm{V}$
$\mathrm{G}$
$\mathrm{G}$
$\mathrm{N}$
$\mathrm{e}$
$\mathrm{t}$
$\mathrm{R}$
$\mathrm{e}$
$\mathrm{s}$
$\mathrm{N}$
$\mathrm{e}$
$\mathrm{t}$

Regularly these systems are pre-prepared to perform arrangement on an enormous picture dataset, for example, ImageNet.

\subsection{Accuracy In Object Detection With Opencv}

Object finder execution is assessed utilizing mean Average Precision (mAP) which depends on the Intersection over Union (IoU) over all classes in our dataset. 
IoU is utilized along with mean Average Precision (mAP) for assessment of the exactness of a thorough learning object indicator. IoU and $\mathrm{mAP}$ can be utilized together to assess the presentation of HOG + Linear SVM indicators, and weighty learning-based methods. Algorithms that give anticipated bouncing boxes (and alternatively class names) as yield can be assessed utilizing IoU. mean Average Precision (mAP) For technicians and professionals who are new to question identification can discover the mAP counts confounding. This is in part because of the way that $\mathrm{mAP}$ is a progressively confounded assessment metric. Figuring the Average Precision (AP) for a specific article recognition pipeline is a three stage process:

1. Register the exactness which is the extent of genuine positives.

2. Register the review which is the extent of genuine positives out of every conceivable positive.

3. Normal together the most extreme exactness esteem over all review levels in steps of size s.

\section{Concepts}

The point of this exploration work is to give an answer utilizing profound learning for distinguishing object and evaluating the accuracy with an audio assistance to have the option to give the relating reaction to the individual utilizing it and tell him about the prompt environment. Two significant zones of this undertaking are-

1. Navigational direction

2. Object distinguishing proof as indicated by its characterization

\subsection{Basic Convolutional Neural Networks:}

- A CNN comprises of an information and a yield layer. The concealed layers of a $\mathrm{CNN}$ commonly comprise of convolutional layers, pooling layers, completely associated layers and standardization layers.

- In CNN, the image is filtered pixel shrewd. The picture is then partitioned into little squares with constrained pixels. After this, various layers of $\mathrm{CNN}$ arrange various items and afterward we put it through different classifiers and lastly figure out an estimated percentage level of what the articlecould be.

- This is a great power strategy.

\subsection{R-CNN:}

- $\mathrm{R}-\mathrm{CNN}$ is a superior path than $\mathrm{CNN}$ for object recognition. 
- It made jumping boxes or locale proposition (2000) utilizing particular pursuit.

- It is essentially taking a look at a picture through different windows of different sizes and afterward attempts to gather them by pixels, surfaces, hues or force and goes them through various classifiers to distinguish objects.

- It takes just about 47-50 seconds to give a yield.

\subsection{Fast R-CNN:}

- Fast R-CNN expands on past work to productively order object recommendations utilizing prominent convolutional systems.

- Fast R-CNN utilizes a few advancements to improve preparing and testing speed whileadditionally expanding discovery exactness.

- Fast R-CNN trains the extremely profound system 9x quicker than R-CNN, is $213 x$ quicker at test-time and is more precise than R-CNN.

- The explanation for Fast R-CNN being quicker than R-CNN is on the grounds that we don't encourage 2000 area recommendations to the CNN inevitably. Rather, the convolution activity is done just once per picture and a component map is produced from it.

\subsection{Faster R-CNN:}

- Both of the above calculations utilize particular search to discover the area proposition.

- It is an ineeficient procedure influencing the exhibition of the system.

- Therefore, certain researchers thought of an item identification calculation disposing of the specific inquiry calculation and enabling the system to become familiar with the district.

\section{Proposed System}

A framework should be created which can be a manual for all outwardly hindered individuals to help them inside and out in order to make them mindful of what is happening, by what method should one respond and so forth. We use AI and profound learning in planning an ongoing application that gives constant imaging and handling what is front of the individual and the distance of the item. At first we need to get ongoing video recording which is passed onto server for substantial handling by means of calculations and perform object recognition to comprehend 
what the item is. There will likewise be a navigational guide which will help with the distance of the item from the stick where camera is introduced. Analysis of all this will result in an output as $3 \mathrm{D}$, sound will be produced in the earpiece which the user should wear. This research plans to utilize most recent and quickest calculation accessible and train our model with enormous dataset for it. This entire framework plans to carry solace and easiness in the lives of such individuals. This examination won't just add to the integrity of society also it will be an extraordinary experience of learning.

\subsection{Deep Learning For Object Detection}

1. An info picture is exhibited at first and a class name is acquired.

2. But while object discovery is performed, we can display an info picture and getnumerous bounding boxes and class names out.

3. Then standard, PC vision based article location strategies are applied.

4. The pre-prepared system is taken and utilized as a base system in a profound learningobject discovery structure (i.e., Faster R-CNN, SSD, YOLO).

\subsection{Caffe Model:}

CAFFE is a totally unique methodology.

CAFFE in reality just takes a gander at the image just once.

It partitions the picture in a lattice of $13 \times 13$ boxes. Each case is liable for anticipating 5 bouncing boxes.

A bouncing box depicts the square shape that encases an article.

It likewise computes a certainty score that reveals to us how certain it is that the container contains an item.

It fills in as a classifier and predicts the class of the item. See Figure 1.

If the certainty score is more than 30 (threshold worth) at that point we keep the case since it is probably going to encase an item.
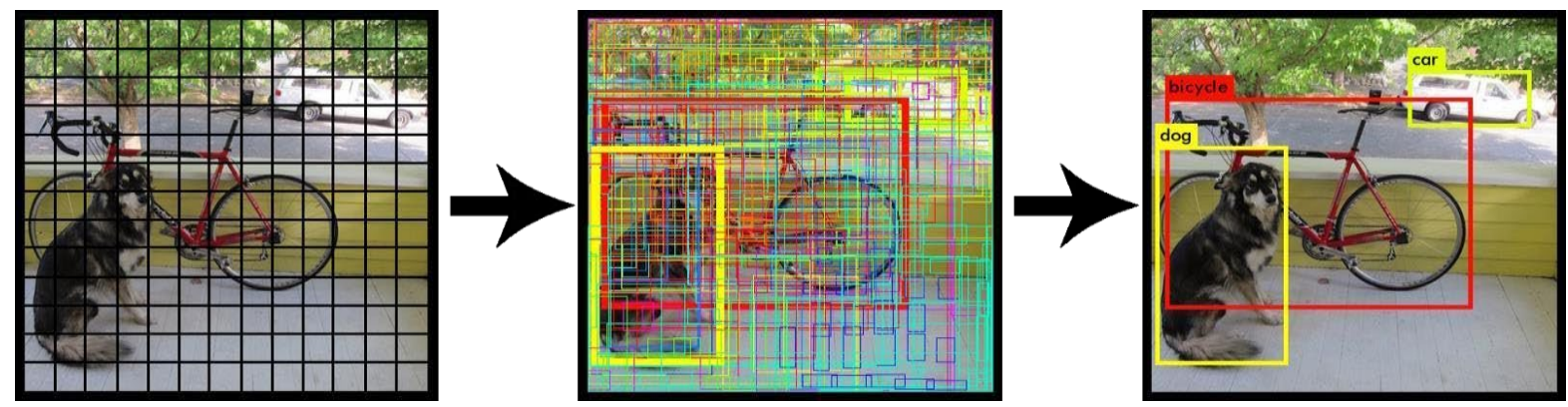
Fig 1 Object identification utilizing CAFFE

Model

4.2.1 Voice Assistance: For voice help, we can utilize Python Text to discourse, otherwise called pyttsx3. We can ask the PC to communicate in with programming language Python. It expresses the composed words in the English language. pyttsx 3 is a module and order line utility to spare spoken content to $\mathrm{mp} 3$. The yield from the CAFFE model and the profundity estimation calculation will be passed to the python code of pyttsx3 code. The yield will be a voice training the visually impaired individual about the article.

\section{Result and Discussion}

\subsection{Goals of proposed framework}

1. Precision: - The degree of exactness in the proposed framework is high. All work would be done effectively and it guarantees that whatever data is originating from the focal handling on servers isexact

. The model guarantees high precision and execution.

2. Unwavering quality: - The dependability of the proposed framework will be high. The explanation behind the expanded dependability of the framework is that we can store the calculation repetitively as though one bombs the other server consequently takes over until the past one is fixed. So thereis nothing to stress over reinforcements.

3. Yield consistency - As the model is prepared through a dataset which is exceptionally dependable and can't be prepared again by means of some malevolent client, the yield conveyed will consistently be reliable and genuine.

4. Simple to Operate - The framework structured ought to be anything but difficult to work and oughtto be to such an extent that it very well may be refreshed effectively and inside a brief timeframe and it should effortlessly fit in the restricted spending plan of the client.

\subsection{Flowchart:}




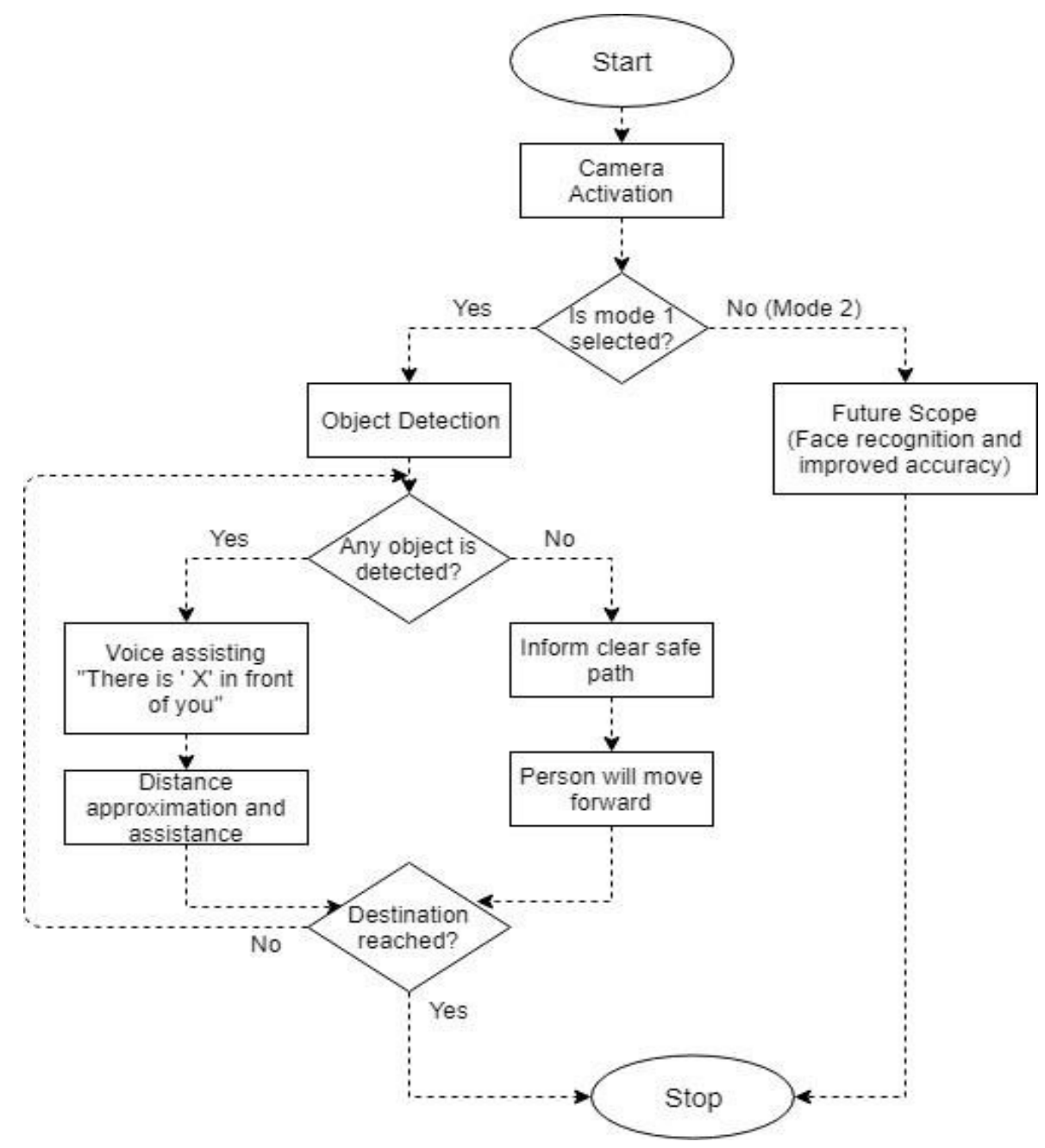

The procedure can be portrayed as when an outwardly debilitated individual start to utilize this gadget, at first the camera is enacted and a mode is chosen. Here the mode 1 allude to objects location and navigational direction at better places of concern. Utilizing mode 2 is past extension starting at now and is reached out in significant task. On the off chance that mode 1 is chosen a steady reconnaissance distinguishes whether there is an item before the camera. Assuming no, voice help will manage an unmistakable, safe way. In the event that indeed, Voice associate will provoke "There is ' $\mathrm{X}$ ' object before you. 


\subsection{Problems Faced}

\section{a) Overlap}

Covering objects and bounding boxes hamper the tried item in the identification pipeline. Most importantly, covering of the genuine items can make trouble in putting the bounding boxes unambiguously. The bounding boxes are chosen by the region generation technique and, ordinarily, they ought to encompass the total item. In case, the item is impeded, we can't know the elements of the article before we have characterized it.

It relies upon the application whether it is critical to recognize different cases of a similar article near one another in any case. A self-driving vehicle should begin breaking when it identifies the nearness of an individual in the street. For this situation, it isn't pivotal to know promptly whether the identification comprises of a couple of people or not. On the other hand, if the assignment is to include the quantity of members in an open occasion, we should have the option to unquestionably differentiate individuals.

\section{b) Built-in invariance}

A principal issue of $\mathrm{PC}$ vision is figuring out how to isolate the properties of the picture from the inborn properties of the article itself. Essentially, this implies the article identifier must be invariant to shifts in perspective, lighting, clamor and different conditions brought about by the properties and arrangement of the camera, as opposed to the item itself. We have demonstrated that utilizing profound learning systems we can obtain a large portion of this invariance consequently from the initial information, given that the given information is adequately varied. Along these lines, it is data dependent, to show which invariance types are valuable to learn.

One of the strategies created to empower preparing of more profound systems is called cluster standardization. The point of the technique is to keep the appropriation of the layer inputs stable during preparing. Putting it generally, it is useful to process the neural system input initially to have zero mean and a unit difference. In cluster standardization, rather than performing standardization once first and foremost, it is likewise performed during preparation and between layers. Batch standardization empowers in getting a higher learning rate.

\section{c) Depth of Network}

The strategy in its present structure doesn't improve the exhibition of a convolutional object indicator, with certain minimal cases as exception. It is hard to isolate cases from the various situations where the technique corrupts execution. From a commonsense perspective, the 
strategyis non-productive, as it requires a long calculation time, which would have made it unreasonable regardless of whether it had proceeded true to its form. The negative outcomes from the geometric induction can be seen as a disdain of the exhibition abilities of best in class frameworks. Quick R-CNN as of now functions admirably enough to render insignificant the impacts of a framework intended for the previous object identifiers, and as we have illustrated, numerous strategies exist for improving the recognition speed and exactness of Fast R-CNN.

\section{Conclusion}

In this exploration venture, we research the need for visually hindered individuals. In light of the driving force of the $\mathrm{CNN}$, we give an answer to build up a visually impaired perception framework that causes blind individuals to investigate the encompassing condition in an improved way. A versatile and ongoing arrangement is given in the above paper. We present a platform that uses convenient and efficient cameras, quick HD video connect and incredible server to empower google voice assistance. By utilizing CAFFE calculation which tells the most elevated likelihood of the article to be something out of state four other closest conceivable outcomes of that item and propelled remote transmitter (Bluetooth) the arrangement could perform exact ongoing item location with live stream. A model for tactile substitution (vision to hearing) can be set up effectively. Through this examination, we would like to exhibit the plausibility of utilizing PC vision methods as a sort of assistive innovation to facilitate the everyday existence of such individuals.

\section{Acknowledgements}

This examination paper was done under Dr. Sapna Jain, Assistant Professor and Project Supervisor of Jamia Hamdard University, New Delhi, and she upheld me with her understanding and information regarding the matter, which pervaded me with the inclination to work diligently.

\section{References}

[1]. What Is Deep Learning? | How It Works, Techniques and

Applications. Accessible:

https://www.mathworks.com/revelation/profound learning.html [Jun 8, 2018].

[2] Prof. Seema Udgirkar, Shivaji Sarokar, Sujit Gore, Dinesh Kakuste and Suraj Chaskarresearch paper 
[3]. An Intuitive Explanation of Convolutional Neural Networks.

2016. Accessible: https://ujjwalkarn.me/2016/08/11/natural

clarification convnets/[Jun 12, 2018].

[4] Simonyan, K., Zisserman, A.: Very deep convolutional networks for large-scale image recognition. arXiv preprint arXiv:1409.1556 (2014)

[5]. ROSEBROCK, An., Object location with profound learning and OpenCV. 2017.

Accessible: https://www.pyimagesearch.com/2017/09/11/object-location with-profound

learning-andopencv/[Jun 13, 2018

[6] A Review Paper on Object Detection for Improve the Classification Accuracy and Robustness using different Techniques by Divya Patel Research Scholar, Parul Institute of Technology and Pankaj Kumar Gautam Parul Institute of Technology.

[7] Image Processing and Object Detection By Nidhi, NIT Kurukshetra, Haryana, India.

[8] Object Detection Combining Recognition and Segmentation By Liming Wang , Jianbo Shi, Gang Song and I-fan Shen

[9] A. Quattoni, and A.Torralba. Recognizing Indoor Scenes. IEEE Conference on Computer Vision and Pattern Recognition (CVPR), 2009.

[10] M. Hebert, J. Ponce, T. Boult and A. Gross (Eds.). Object Representation in Computer Vision.

Springer-Verlag, Berlin, 1995

[11] Object Detection by Yali Amit and Pedro Felzenszwalb, University of Chicago

[12] https://arxiv.org/abs/1506.01497

[13] Faster R-CNN: Towards Real-Time Object Detection with Region Proposal Networks by Shaoqing Ren, Kaiming He, $\underline{\text { Ross B. Girshick, Jian Sun }}$

[14] Study on Different Region-Based Object Detection Models Applied to Live Video Stream and Images Using Deep Learning by Jyothi Shetty \& Pawan S. Jogi

[15] Receptive Field Block Net for Accurate and Fast Object Detection by Songtao Liu, Di Huang, and Yunhong Wang; The European Conference on Computer Vision (ECCV), 2018, pp. 385-400

[16] R-CNN for Small Object Detection by Chenyi Chen, Ming-Yu Liu, Oncel Tuzel, Jianxiong Xiao 
[17] Simonyan, K., Zisserman, A.: Very deep convolutional networks for large-scale image recognition. arXiv preprint arXiv:1409.1556 (2014) 